\title{
MicroCT image based simulation to design heating protocols in magnetic nanoparticle hyperthermia for cancer treatment
}

\author{
Alexander LeBrun, Ronghui Ma, and Liang Zhu \\ Department of Mechanical Engineering \\ University of Maryland Baltimore County \\ Baltimore, MD 21250
}

Corresponding author:

Liang Zhu, Ph.D.

Professor of Mechanical Engineering

University of Maryland Baltimore County

1000 Hilltop Circle

Baltimore, MD 21250

Tel: 410-455-3332

Email: zliang@umbc.edu 


\begin{abstract}
Objectives: The objective is to design heating protocols to completely damage PC3 tumors after a single magnetic nanoparticle hyperthermia session with minimal collateral thermal damage, based on microCT image generated tumor and mouse models. Methods: Tumor geometries and volumetric heat generation rate distributions that are generated from microCT scans in our previous study are imported into COMSOL 4.3® multiphysics for heat transfer simulations and heating protocol design using the Arrhenius damage model. Then, parametric studies are performed to evaluate how significantly the infusion rate affects the protocol design and its resulted collateral thermal damage. Results: The simulated temperature field in the generated tumor geometry and volumetric heat generation rate distribution are reasonable and correlates well with the amount of the total thermal energy deposited into the tumors. The time needed for complete thermal damage is determined to be approximately 12 minutes or 25 minutes if one uses the Arrhenius integral $\Omega$ equal to 1 or 4 as the damage threshold, when the infusion rate is $3 \mu \mathrm{L} / \mathrm{min}$. The heating time increases $26 \%$ or $91 \%$ in the higher infusion rate groups of 4 or $5 \mu \mathrm{L} / \mathrm{min}$, respectively. Collateral thermal damage to the surrounding tissue is also assessed. Although the two larger infusion rate groups can still cause thermal damage to the entire tumor, the collateral thermal damage would have exceeded the design criterion of 5\%, while the assessment criterion is acceptable only in the infusion rate group of $3 \mu \mathrm{L} / \mathrm{min}$. Based on the results of this study, we identify an injection strategy and heating protocols to be implemented in future animal experiments to evaluate treatment efficacy for model validation.
\end{abstract}

Key words: magnetic nanoparticle hyperthermia, injection strategy, image-based simulation, heating protocol design, bioheat transfer 


\section{Introduction}

Currently, the main treatment options for cancer are surgery, radiation, and chemotherapy. Although those traditional interventions have increased the five-year survival rate in cancer patients, none of them have been able to consistently kill or remove all cancerous tissues at the original site. The remaining tumor cells may contribute to tumor recurrence and/or cancer metastasis. This suggests a need to develop an effective treatment strategy to eliminate all cancerous tissues while ensuring safety of the surrounding healthy tissue.

In the past decade, it has been demonstrated that magnetic nanoparticles can deliver confined thermal energy to tumors when subject to an alternating magnetic field, therefore holding a high cell-killing potential while minimizing collateral thermal damage to the surrounding tissue in cancer treatment [Ito et al., 2005; Johannsen et al., 2005; 2007; Jordan et al., 2006; Wust et al., 2006; Maier-Hauff et al., 2007; Zhao et al., 2012]. Existing studies also suggest that nanoparticle distribution in tumors is a major factor in determining treatment efficacy [Wust et al., 2006; Johannsen et al., 2007; Attaluri et al., 2011]. Recent experimental studies using microCT and other imaging techniques have provided strong evidence that nanoparticle distributions in tumors are often unpredictable [Kalambur et al., 2005; Johannsen et al., 2007; Attaluri et al., 2011; LeBrun et al., 2013; Wabler et al., 2014]. In systemic delivery of nanoparticles to tumor sites, vascular permeability, local blood perfusion distribution, and particle penetration in the tumor region are often difficult to quantify [Kong and Dewhirst 1999; Urono et al., 1999; McGuire et al., 2001; Liu et al., 2005; Dreher et al., 2006]. In intratumoral injections to tumors, nanoparticle deposition in tumors is mainly determined by diffusion and advection of the carrier solution in heterogeneous microstructure of tumors. Unfortunately, particle distribution in tumors is difficult to predict due to the heterogeneous tumor porosity, 
micro-crack formation during injection, and the random nature of particle interactions with cells in the extracellular matrix such as particle deposition on the cell surface, intercellular uptake, and particle aggregation. In addition, tumors in clinical settings may have various sizes and shapes, which result in different nanoparticle distribution from one tumor to another. It is therefore, important to design individualized heating protocols based on image-generated tumor geometry and nanoparticle distribution so that the spatial tumor temperature elevations can be precisely controlled in hyperthermia treatment. The image-based design approaches need to be verified via carefully designed in vivo animal studies before applied to clinical settings.

We have shown in a previous study that microCT imaging technology can be utilized to determine nanoparticle distribution volume in opaque PC3 tumors after injecting 0.1 cc commercially available ferrofluid at the tumor center [LeBrun et al., 2015]. In that study, the nanoparticle distribution volume is used to evaluate how far the nanoparticles spread by average from the injection site of the tumor. It has been shown that a low intratumoral injection rate minimizes variation of the nanoparticle distribution volume. Therefore, an injection strategy has been identified to result in relatively repeatable and controllable nanoparticle distribution patterns in PC3 tumors. It is expected that one will obtain similar nanoparticle distribution volume in the same type of tumors, if one implements the same injection strategy. In addition, the microCT imaging system has been utilized to generate tumor geometries and volumetric heat generation rate distribution, setting the foundation for designing individualized treatment protocols.

Accuracy of simulated temperature distributions in tumors induced by magnetic nanoparticles relies on precise description of tumor geometry and its surroundings, tissue thermal and physiological properties, and local concentration distribution of nanoparticles. Therefore, 
realistic and accurate models of the tumor geometry and nanoparticle distribution are imperative to guide clinicians to achieve effective and safe treatment through development of individualized heating protocols. In this study, tumor geometries and volumetric heat generation rate distributions generated in our previous study [LeBrun et al., 2015] are imported into COMSOL $4.3 \circledR$ multiphysics (Stockholm, Sweden) for heat transfer simulations and heating protocol design. The goal of this study is to estimate the time it takes to completely damage the tumor after a single heating session and to evaluate the extent of collateral thermal damage. Then, parametric studies are performed to evaluate how significant the infusion rate affects the protocol design and resultant collateral thermal damage.

\section{Methods and Materials}

\subsection{Importing Tumor Geometry, Mouse Model, and Heat Generation Rate Distribution}

The tumor geometry generated in our previous study [LeBrun et al., 2015] was imported into COMSOL 4.3® multiphysics via the Pro/Engineer LiveLink ${ }^{\mathrm{TM}}$ interface. Using the built-in tools of COMSOL, the tumor was scaled to the appropriate size and properly oriented to coincide with the imported files. Then a mouse model generated in Pro/Engineer based on microCT scans of a mouse body was used as a mounting surface for the tumor [Manuchehrabadi et al., 2013]. The mouse model was smoothed and processed using the same tools as that to generate the tumor geometry, allowing for meshing while maintaining the original volume and shape. The hind and forepaws, and the tail were simplified in the model to avoid any singularities during the meshing process. The tumor was mounted in a way so that the flat area of the tumor created during resection was the contact surface with the mouse body. Figure 1 illustrates views of a tumor implanted on a mouse body imported to COMSOL for heat transfer simulation. 
As described in our previous study, the ferrofluid infusion rate strongly influences the resulted nanoparticle distribution in the PC3 tumors. In this study, three volumetric heat generation rate distributions representing possible nanoparticle distribution in the three infusion rate groups were evaluated and tested. For each infusion rate, its volumetric heat generation rate distribution was imported into COMSOL as a source file. Note that the center of the coordinate system was adjusted so that it was consistent to that in the COMSOL geometry of the mouse model. Linear interpolation and constant extrapolation were used between elements to achieve a smooth volumetric heat generation rate distribution as close to the original volumetric heat generation distribution as possible without incurring singularities.

\subsection{Heat Transfer Modeling in COMSOL}

The Pennes bioheat equation [Pennes 1948] was employed in the modeling of the transient temperature elevations in tissue. This is a continuum model that has been used extensively in modeling thermal effect of local blood perfusion in biological tissue [Zhu 2010]. The Pennes bioheat equation was applied to both the mouse body and tumor, shown as:

$$
\begin{gathered}
\rho_{t} c_{t} \frac{\partial T_{t}}{\partial t}=k_{t} \nabla^{2} T_{t}+\omega_{t} \rho_{b} c_{b}\left(T_{b}-T_{t}\right)+Q_{m e t, t} \\
\rho_{c} c_{c} \frac{\partial T_{c}}{\partial t}=k_{c} \nabla^{2} T_{c}+\omega_{c} \rho_{b} c_{b}\left(T_{b}-T_{c}\right)+Q_{m e t, c}+Q_{M N H}
\end{gathered}
$$

where $k$ is thermal conductivity $(\mathrm{W} / \mathrm{m} \cdot \mathrm{K}), \rho$ is density $\left(\mathrm{kg} / \mathrm{m}^{3}\right), c$ is specific heat $(\mathrm{J} / \mathrm{kg} \cdot \mathrm{K}), T_{b}$ is the prescribed arterial blood temperature equal to $37^{\circ} \mathrm{C}, \omega$ is the local blood perfusion rate $(1 / \mathrm{s})$, and $Q_{m e t}$ is the local metabolic heat generation rate $\left(\mathrm{W} / \mathrm{m}^{3}\right)$. The subscripts $t, b$, and $c$ denote tissue, blood, and tumor tissue, respectively. The nanoparticle induced volumetric heat generation rate $\left(\mathrm{W} / \mathrm{m}^{3}\right)$, or $Q_{M N H}$, was only applied to the tumor region since it is assumed that 
the nanoparticles are confined into the tumor region. Thermal and physiological properties used in the model are presented in Table 1. It was assumed that the thermal and physiological properties remained constant and isotropic within each domain to simplify the model [Rylander et al., 2006; Trakic et al., 2006]. The temperature dependency of the tumor blood perfusion rate was considered in this study [Lang et al., 1999], and shown as:

$$
\omega_{c}=\left\{\begin{array}{lr}
0.833 \times 10^{-3} & T<37^{\circ} \mathrm{C} \\
0.833 \times 10^{-3}-\frac{(T-37)^{4.8}}{5.438 \times 10^{3}} & 37^{\circ} \mathrm{C} \leq T \leq 42^{\circ} \mathrm{C} \\
0.416 \times 10^{-3} & T>42^{\circ} \mathrm{C}
\end{array}\right\}\left(\frac{1}{s}\right)
$$

As the temperature increases, the tumor blood perfusion rate decreases. The temperature dependency of the metabolic heat generation rate was not considered since the effect has not been well documented for the temperature range in this study.

\subsection{Boundary Conditions}

The mouse body was considered to be exposed to a natural convection condition where the ambient air temperature was prescribed as $25^{\circ} \mathrm{C}$ and the heat transfer coefficient was 10 $\mathrm{W} / \mathrm{m}^{2} \cdot \mathrm{K}$ [Manuchehrabadi et al., 2013]. The bottom of the mouse model was prescribed as a uniform temperature of $37^{\circ} \mathrm{C}$ to simulate the effect of the heating pad. A convective boundary condition was also applied to the tumor using a correlation for a sphere:

$$
\begin{gathered}
h=\frac{N u_{D, a v} k_{\text {air }}}{D} \\
N u_{D, a v}=\left\{0.60+\frac{0.387 R a_{D}^{1 / 6}}{\left[1+(0.59 / P r)^{9 / 16}\right]^{8 / 27}}\right\}^{2}
\end{gathered}
$$

where $N u_{D, a v}$ is the Nusselt number with respect to the average diameter of the tumor, $h$ is the heat transfer coefficient, $D$ is the average diameter of the tumor, $k_{\text {air }}$ is the thermal conductivity 
of air at $25^{\circ} \mathrm{C}(0.028 \mathrm{~W} / \mathrm{m} \cdot \mathrm{K}), R a_{D}$ is the Rayleigh number, and $\operatorname{Pr}$ is the Prandtl number with a value of 0.707 . This approximation was used since the heat transfer coefficient for the exact tumor geometry would be difficult to determine. PC3 tumors tend to favor more hemispherical growth [Rylander et al., 2006]. Typical values of the heat transfer coefficient $h$ calculated from this study ranged from $3.7-4.2 \mathrm{~W} / \mathrm{m}^{2} \cdot \mathrm{K}$, depending on the size of the tumor.

\subsection{Numerical Simulation Parameters}

The mesh was generated by COMSOL 4.3 and it was finer inside the tumor with a growth factor of 1.4 starting from the tumor boundary towards the normal tissue. The maximum element size was $0.008 \mathrm{~m}$ while the minimum element size was $0.0005 \mathrm{~m}$. The total number of the tetrahedral elements using the fine mesh setting was 146,062. The mesh sensitivity was checked via increasing the mesh setting to extremely fine meshing with an increase in the total number of elements to 644,035. The four-fold increase in the mesh elements resulted in a difference of less than $0.1^{\circ} \mathrm{C}$ in the average temperature in the tumor. The temporal resolution was selected as 0.01 seconds. Lowering the temporal resolution by half resulted in a less than $0.1 \%$ change in the average tumor temperature.

\subsection{Thermal Damage Assessment}

Thermal damage in tissue was assessed by a first-order thermal-chemical rate equation coupled with the Pennes bioheat equation simulating the temperature field. Although it is widely recognized that tissue injury is the result of complicated reaction mechanisms, progression of thermal injury can be considered as a uni-molecular process where native molecules are transformed into a coagulated/denatured state leading to cell death [Marqa et al., 2011; Dewhirst et al., 2015]. The temperature-time history determines the extent of thermal damage at a specific location $(x, y, z)$. It is quantified using a parameter, $\Omega$, calculated from the Arrhenius equation: 


$$
\Omega(x, y, z, \tau)=\ln \left[\frac{\varsigma(0)}{\varsigma(\tau)}\right]=A \int_{0}^{\tau} e^{-\frac{E_{a}}{R_{u} T_{t}(x, y, z, t)}} d t
$$

where $\varsigma(0)$ is the initial concentration of healthy cells, $\varsigma(\tau)$ is the concentration of healthy cells remaining after heating of a duration $\tau$ (seconds), $A$ is the frequency factor $(1 / \mathrm{s}), E_{a}$ is the activation energy $(\mathrm{J} / \mathrm{mol}), R_{u}$ is the universal gas constant $(8.23 \mathrm{~J} / \mathrm{mol} \cdot \mathrm{K})$, and $T_{t}(x, y, z, t)$ is the absolute tissue temperature at a given location. The values for the frequency factor and activation energy are cell line and tissue dependent. In this study, the temperature dependency of the frequency factor and activation energy for PC3 cells was from a previous study and listed in Table 2. Before treatment, $\Omega$ is zero, it then increases with heating. Based on Equation 5, 63\% and 99\% of the cells are damaged (denaturation of proteins occurs), when $\Omega$ is 1 and 4 , respectively.

\subsection{Criteria for Designing Treatment Protocols}

One of the features of COMSOL software is its multiphysics platform. In this study, calculation of the thermal damage parameter, $\Omega$, is implemented in the COMSOL software to solve for the discretized form of Equation 5, and this equation can be solved simultaneously with the temperature field in the tumor and its surrounding tissue to determine the progression of the thermal damage distribution with time.

The goal for designing treatment protocols of hyperthermia therapy is to predict the extent of heating-induced cell death. An ideal thermal therapy for cancer treatment is to induce irreversible damage to the cancerous cells to prevent tumor recurrence or metastasis, and to preserve the surrounding healthy tissue. In order to identify a treatment protocol, two criteria are proposed to be satisfied in this study: $100 \%$ damage to the tumor cells and a ratio of the damaged normal tissue to the tumor volume less than 5\%. Previous studies usually used a percentage of 
damage in the normal tissue to a defined normal tissue volume [Manuchehrabadi and Zhu 2014]. It seems that the definition of normal tissue volume is very arbitrary and subjective in previous studies. Since there is no established standard for an acceptable amount of collateral thermal damage, our damage criterion is defined as the ratio of the damaged normal tissue to the known tumor volume. The percentage will be calculated by the following equation:

$$
\% V_{\text {damage }}=\left(\frac{V_{\text {damaged normal tissue }}}{V_{\text {tumor }}}\right) \times 100 \%
$$

where $V_{\text {damaged normal tissue }}$ is the volume of thermally damaged healthy tissue in the mouse body and $V_{\text {tumor }}$ is the volume of the tumor.

As shown in Equation 5, thermal damage depends on two major parameters: (1) the duration of heating, and (2) the temperature elevation. Since the magnetic field strength and the type and amount of the injected ferrofluid are fixed [LeBrun et al., 2015], the only variable in this study is the duration of heating. The heating time needed to achieve a minimum of $\Omega=4$ in the entire tumor will be identified as the treatment time. In addition, a heating duration to achieve a minimal $\Omega=1$ will also be explored to determine whether or not $63 \%$ denaturation of proteins would be sufficient enough to cause irreversible thermal damage without tumor recurrence. If the percentage of tissue damage in the healthy region is less than $5 \%$ of the volume of tumor, the identified heating time is acceptable; otherwise, cooling in the normal tissue region and/or lowing the maximal temperature in the tumor may be needed.

\section{Results}

\subsection{Temperature Elevations in the Tumor and Its Surroundings}


Figure 3 gives one slice of a reconstructed cross-sectional microCT image when the infusion rate is $3 \mu \mathrm{L} / \mathrm{min}$, the $Q_{M N H}$ distribution contour, and steady-state temperature distribution contour in the COMSOL. The similarity between the microCT images and the $Q_{M N H}$ distribution in the COMSOL suggests the confidence of the $Q_{M N H}$ data transfer process from the microCT scans to the COMSOL software package. It is also observed that the magnitude of the $Q_{M N H}$ is in an expected range. The location of the maximal temperature rise in the tumor coincides with the region containing most nanoparticles and the rest of the tumor tissue is heated by heat conduction.

Figure 4 illustrates the steady-state temperature distribution in a tumor mounted on a mouse body at various time instants. The temperature field in the mouse body before or during the heating is relatively uniform, varying from approximately $35^{\circ} \mathrm{C}$ on its boundary surfaces to $37^{\circ} \mathrm{C}$ towards the center. Most temperature elevations occur in the tumor region due to nanoparticle-generated heating. The interface between the mouse body and the tumor has illustrated significant heat removal from the hot tumor region to the warm mouse body. The steady-state tumor temperature field is established after heating of approximate15 minutes.

\subsection{Designing Heating Protocols for Individual Tumors}

Once the transient temperature distribution is determined, it is then used in the Arrhenius integral to determine the distribution of the damage parameter, $\Omega$. Figure 5 shows the damages on one cross-sectional plane of a tumor before treatment and after 5, 12, 15, 20, and 25 minutes of heating, respectively. The void region represents the damaged tumor region when $\Omega \geq 4$. It can be clearly seen that before the steady-state temperature field is established, not much damage occurs in the tumor. As the heating time increases and heat conducts throughout the tissue, more and more damage in the tumor is observed. Our results have shown that it takes more than 25 
minutes to completely damage the entire tumor (Figure $5 f$ ). Figures $5 \mathrm{~b}$ and $5 \mathrm{c}$ are also used to estimate the heating time if $\Omega \geq 1$ is used to define thermal damage. The tumor region is separated by a heavy white line representing the tumor locations with $\Omega=1$. One notices that the heavy white line is located at the bottom boundary of the tumor when the heating time is approximately 12 minutes (Figure 5c), implying a heating duration of 12 minutes is sufficient to induce $\Omega \geq 1$ in the entire tumor.

To verify whether the prescribed heating duration for inducing complete thermal damage to the tumor also satisfies the imposed $2^{\text {nd }}$ criteria, the volume of tissue in the mouse body with a value greater that $\Omega=4$ is determined. Based on the damage contours in Figure 6, it is clear that collateral thermal damage to the surrounding healthy tissue is noticeable at the end of the heating duration of 25 minutes.

Simulations are performed for one tumor in each infusion rate to evaluate how the infusion rate affects the heating protocol design. Figure 7 compares the heating time when using either $\Omega \geq 1$ or $\Omega \geq 4$ as a definition for irreversible thermal damage in tumors in the three infusion groups. Heating time required to achieve $\Omega \geq 1$ is approximately half of that when the definition of damage is $\Omega \geq 4$. When $\Omega \geq 4$ is used as the definition of damage, the heating duration is about 1500 seconds ( 25 minutes) if the infusion rate is $3 \mu \mathrm{L} / \mathrm{min}$. As shown in Figure 7, the heating time increases to 1900 seconds when the infusion rate is $4 \mu \mathrm{L} / \mathrm{min}$, further to 2900 seconds when the infusion is $5 \mu \mathrm{L} / \mathrm{min}$.

\subsection{Evaluation of Thermal Damage in Healthy Tissue}

Collateral thermal damage in the healthy tissue is inevitable. It has been shown that a healthy tissue volume of $55 \mathrm{~mm}^{3}$ is damaged in the infusion group of $3 \mu \mathrm{L} / \mathrm{min}$, the collaterally 
damaged healthy tissue volumes are much bigger in the larger infusion rate groups of $4 \mu \mathrm{L} / \mathrm{min}$ $\left(78 \mathrm{~mm}^{3}\right)$ and $5 \mu \mathrm{L} / \min \left(141 \mathrm{~mm}^{3}\right)$. Since the volume of all the tumors used in the study varies $\left(1013 \pm 114 \mathrm{~mm}^{3}\right)$, it is important to evaluate the percentage of the volume of collateral thermal damage to the healthy tissue to the tumor volume. Showing in Figure 8, the percentage is 4.8\%, $8.6 \%$ and $14.1 \%$ for the infusion rate of $3 \mu \mathrm{L} / \mathrm{min}, 4 \mu \mathrm{L} / \mathrm{min}$, and $5 \mu \mathrm{L} / \mathrm{min}$, respectively. The percentage in the infusion group of $3 \mu \mathrm{L} / \mathrm{min}$ is below our imposed criterion of $5 \%$, while the collateral damage percentages in the other two infusion groups are above the imposed criterion. Therefore, only the results obtained for the infusion group of $3 \mu \mathrm{L} / \mathrm{min}$ satisfy our two design criteria.

\section{Discussion}

Our study illustrates that a heat transfer model based on microCT image-generated tumor geometry and volumetric heat generation rate distribution should greatly improve model predicting power, and the approach has the potential of developing individualized treatment designs for various tumor sizes and types in the future. The theoretical simulation results suggest that the nanoparticle deposition distribution in the infusion rate group of $3 \mu \mathrm{L} / \mathrm{min}$ not only results in the shortest heating time to cause irreversible thermal damage to tumors, but also leads to the smallest amount of collateral damage to the surrounding healthy tissue. The predicted transient temperature fields and thermal damage assessments imply that an injection strategy of using the $3 \mu \mathrm{L} / \mathrm{min}$ satisfies the two designing criteria. On the other hand, although the other two larger infusion rate groups can still cause thermal damage to the entire tumor, the heating time is at least $26 \%$ longer to achieve the goal and the collateral thermal damage would have exceeded the designed criterion of $5 \%$. 
Thermal dosage needed in a tumor depends on the size and shape of the tumor, boundary conditions, the extent of the average spreading of nanoparticles from the injection site, the shape of the nanoparticle distribution region, and the total heat generation rate in the tumor. The infusion rate is an important factor affecting the heating time, since it plays a crucial role in determining the average spreading of the nanoparticles from the injection site. From the results obtained in the theoretical simulations, it can be seen that the average heating time for the tumors decreases from $2900 \mathrm{~s}$ in the group of $5 \mu \mathrm{L} / \mathrm{min}$ to $1500 \mathrm{~s}$ in the group of $3 \mu \mathrm{L} / \mathrm{min}$, resulting in a drop of $49 \%$ in the heating time. A higher infusion rate results in a larger nanoparticle distribution volume and/or more nanoparticle accumulation at tumor periphery [Flessner et al., 2005; Attaluri et al., 2011; LeBrun et al., 2015], therefore, heating is more spread in the higher infusion rate groups. This leads to higher temperatures at the tumor surface, possibly resulting in more convection heat loss to the cold ambient environment. It is believed that more heat loss to the air environment in the higher infusion rate groups also results in lower temperatures at the tumor-mouse body interface. Examining the 3-D temperature contours in the tumor and its surrounding mouse body, one notices that the predicted minimal tumor temperature at the tumormouse body interface is lower in the higher infusion rate groups, therefore, requiring a longer heating time to cause irreversible thermal damage at the interface. If the objective of a designed heating protocol is to achieve $100 \%$ thermal damage to tumors, the tumor location where the minimal temperature occurs is of special importance in assessment of tumor damage. Theoretical simulation can be used in the future to guide clinicians on where to place a temperature sensor during heating, therefore, providing real-time monitoring of thermal dosage.

One result obtained in this study is in the collateral thermal damage assessment in surrounding healthy tissue. It is found that larger volumes of the damaged normal tissue are 
associated with higher infusion rates. One reason may be due to the resulted longer heating time required to cause irreversible thermal damage to the entire tumor. A longer heating duration results in more heat spreading from the hot tumor to the warm mouse body, therefore, leading to more collateral damage. Unlike drug delivery to tumors where a more uniform distribution of drug concentration in tumors is more desirable, we believe that in hyperthermia treatment, a more spreading nanoparticle distribution to tumor periphery may induce more collateral thermal damage to surrounding healthy tissue.

Previous investigators have used either $\Omega=1$ or $\Omega=4$ as the minimal threshold for irreversible thermal damage. Our theoretical simulation predicts the heating time for both thresholds. It is expected that using $\Omega=4$ would lead to more than double heating time when comparing to that using $\Omega=1$. When $\Omega=1$ is used as a threshold, there would have been a significant portion of the tumor region that may not be completely damaged. This may later lead to tumor recurrence and/or metastasis. However, future animal/clinical studies are need to evaluate whether $\Omega=1$ as the minimal threshold is still sufficient to damage the entire tumor and to maintain the shrinkage of the tumor for a long period of time. The predicted temperature elevations in the tumors agree with experimental measurements in previous studies. We measured the temperature elevations inside PC3 tumors using a similar magnetic field strength and the same ferrofluid, except that the infusion rate is much larger in our previous study $(5,10$, and $20 \mu \mathrm{L} / \mathrm{min}$ ) [Attaluri et al., 2011]. The measured temperature elevations along a central path of tumors varied greatly from $8^{\circ} \mathrm{C}$ to $35^{\circ} \mathrm{C}$ above the baseline temperature of $37^{\circ} \mathrm{C}$. In this study our simulation suggests that tumor temperature may be elevated $8-41^{\circ} \mathrm{C}$ above its baseline values. The predicted range of temperature elevations falls in the range of the experimental results. The maximum temperature observed in our study also agrees well with those found in 
other clinical and experimental studies [Johannsen et al., 2005; 2007; Maier-Hauff et al., 2011]. Future experimental studies will be performed to provide a more direct comparison to measured temperature elevation in PC3 tumors, following the designed heating protocol.

There are several limitations with the model used in this study. First, the location of the tumor on each mouse was difficult to precisely quantify, although PC3 tumor cells were injected at the same flank location. For simplicity, we only constructed a single mouse model and attach all tumors to it. Although major temperature elevations occur in the tumor region and the temperature distribution in the mouse body is relatively uniform, the variation on the size and shape of the mouse could potentially affect the transient temperature distribution and the total heating time for complete thermal damage.

The temperature dependencies on all thermal and physiological properties were not considered in this study, based on previous studies that properties such as density and heat capacity do not vary much in the temperature range experienced in this study [Rylander et al., 2006]. Another assumption used in this study is that the tissue properties are homogenous. Imaging technology could potentially be used to determine the spatial heterogeneous properties of tissue based on the variations in radiodensity. For this study, the blood perfusion rate inside the tumors was not measured directly during the experiments and an assumed relationship between blood perfusion rates and local temperatures is used. In an ideal situation, there should have been simultaneous measurements of the local blood perfusion rate and temperatures. Not being able to measure them simultaneously is still a limitation of any theoretical simulation of temperature fields in tissue. Finally, although the Arrhenius model has been used in more than half a century, recent investigations have suggested that it overestimates cell death fraction [Pearce, 2015]. A recent paper [Pearce 2015] has proposed a modified Arrhenius model adding 
a "shoulder" region before the typical linear curve. It has been shown that agreement between experimental measurements of cell death and prediction by the Arrhenius model is improved significantly once a temperature-dependent time delay (“shoulder”) is implemented. Future experimental studies should be designed to examine its effect on the thermal dosage and treatment efficacy.

The $Q_{M N H}$ file implemented in COMSOL software package is based on quantification of heat generation in dilute ferrofluid, without taking into consideration of inter-particle interactions. It is not clear how the local temperature elevation may contribute to enhanced nanoparticle interactions. When particles aggregate, their heat generation mechanisms (i.e. relaxation, hysteresis, and eddy-currents) may change to affect the overall amount of heat generation rate [Etheridge et al., 2014]. The calibration methods used to determine the correlation between the nanoparticle concentration and $Q_{M N H}$ were assumed to hold in vivo. Another factor that was not considered in our model is possible changes in the porous structure of the tumors during heating, since the movement of nanoparticles due to increased pore volume during heating may occur. In fact, it has been shown that the nanoparticle distribution volume after heating is bigger than that without heating, suggesting a re-distribution of nanoparticles during the heating [Attaluri et al., 2011]. It has been well documented that hyperthermia can enhance vascular permeability in tumors to facilitate extravasation of drugs to tumor interstitial and further spreading to tumor periphery [Ceelen et al. 2000; Ponce et al. 2006; Zhang et al. 2009; González-Moreno et al. 2010]. The migration of nanoparticles in a porous medium is possible due to diffusion or fluctuation of interstitial pressure. Future experimental studies are warranted to continue to explore this issue. 
In summary, we have designed heating protocols that utilize a volumetric heat generation rate distribution and tumor geometry generated from microCT scans. The simulated temperature field in the generated tumor geometry and simplified volumetric heat generation rate distribution are reasonable and correlate well with the amount of the total thermal energy deposited into the tumors and with results found in previous animal and clinical studies. The time needed for complete thermal damage is determined to be approximately 25 minutes when the infusion rate is $3 \mu \mathrm{L} / \mathrm{min}$, while the heating time is much longer in the higher infusion rate groups. Collateral thermal damage to the surrounding tissue is also assessed and the assessment outcome is only acceptable in the infusion rate group of $3 \mu \mathrm{L} / \mathrm{min}$. Based on the results of this study, we identify an injection strategy and heating protocols to be implemented in future animal experiments to evaluate treatment efficacy for model validation.

\section{Acknowledgement:}

This study is support in part by an NSF research grant CBET-1335958 and the GAANN Scholarship Program at UMBC. The research is performed in partial fulfillment of the requirements for the Ph.D. degree from UMBC by Alexander LeBrun.

\section{References:}

1. Attaluri A, Ma R, Qiu Y, Li W, Zhu L. Nanoparticle distribution and temperature elevations in prostatic tumors in mice during magnetic nanoparticle hyperthermia. International Journal of Hyperthermia 2011; 27(5): 491-502. 
2. Ceelen WP, Hesse U, de Hemptinne B, Pattyn P. Hyperthermic intraperitoneal chemoperfusion in the treatment of locally advanced intra-abdominal cancer. British Journal of Surgery 2000; 87(8): 1006-1015.

3. Dreher MR, Liu W, Michelich CR, Dewhirst MW, Yuan F, Chilkoti A. Tumor vascular permeability, accumulation, and penetration of macromolecular drug carriers. Journal of the National Cancer Institute 2006; 98(5): 335-344.

4. M.W. Dewhirst, J.P. Abraham, B.L. Viglianti, Evolution of Thermal Dosimetry for Application of Hyperthermia Treatment to Cancer, in: Advances in Heat Transfer, Vol. 47, 397-421, 2015.

5. Etheridge ML. Hurley KR, Zhang J, Jeon S, Ring HL, Hogan C et al. Accounting for biological aggregation in heating and imaging of magnetic nanoparticles. Technology 2014; 2(3): 214-228.

6. Flessner MF, Choi J, Credit K, Deverkadra R, Henderson K. Resistance of tumor interstitial pressure to the penetration of intraperitoneally delivered antibodies into metastatic ovarian tumors. Clinical Cancer Research 2005; 11(8): 3117-3125.

7. González-Moreno S, González-Bayón LA, Ortega-Pérez G. Hyperthermic intraperitoneal chemotherapy: rationale and technique. World Journal of Gastrointestinal Oncology 2010; 2(2): 68-75.

8. Haar P J, Broaddus WC, Chen Z, Fatouros PP, Gillies GT, Corwin FD. Quantification of convection-enhanced delivery to the ischemic brain. Physiological Measurement 2010; 31(9): 1075-1089.

9. Ito A, Tanaka K, Honda H, Abe S, Yamaguchi H, Kobayashi T. Complete regression of mouse mammary carcinoma with a size greater than $15 \mathrm{~mm}$ by frequent repeated 
hyperthermia using magnetite nanoparticles. Journal of Bioscience and Bioengineering 2003; 96(4): 364-369.

10. Johannsen M, Gneveckow U, Eckelt L, Feussner A, Waldöfner N, Scholz R et al. Clinical hyperthermia of prostate cancer using magnetic nanoparticles: presentation of a new interstitial technique. International Journal of Hyperthermia 2005; 21(7): 637-647.

11. Johannsen M, Gneveckow U, Thiesen B, Taymoorian K, Cho CH, Waldöfner N et al. Thermotherapy of prostate cancer using magnetic nanoparticles: feasibility, imaging, and three-dimensional temperature distribution. European Urology 2007; 52: 1653-1662.

12. Jordan A, Scholz R, Maier-Hauff K, van Landeghen FKH, Waldöfner N, Teichgraeber U et al. The effect of thermotherapy using magnetic nanoparticles on rat malignant glioma. Journal of Neuro-Oncology 2006; 78: 7-14.

13. Kalambur VS, Han B, Hammer BE, Shield TW, Bischof JC. In vitro characterization of movement, heating and visualization of magnetic nanoparticles for biomedical applications. Nanotechnology 2005; 16: 1221.

14. Kong G, Dewhirst MW. Hyperthermia and Liposomes. International Journal of Hyperthermia 1999; 15(5): 345-370.

15. Lang J, Erdmann B, Seebass M. Impact of nonlinear heat transfer on temperature control in regional hyperthermia. IEEE transactions on Biomedical Engineering 1999; 46(9): 1129-1138.

16. LeBrun A, Manuchehrabadi N, Attaluri A, Wang F, Ma R, Zhu L. MicroCT imagegenerated tumour geometry and SAR distribution for tumour temperature elevation simulations in magnetic nanoparticle hyperthermia. International Journal of Hyperthermia 2013; 29(8): 730-738. 
17. LeBrun A, Joglekar T, Bieberich C, Ma R, and Zhu L. Identification of infusion strategy for achieving repeatable nanoparticle distribution and quantifiable thermal dosage in magnetic nanoparticle hyperthermia. International Journal of Hyperthermia 2015; in press.

18. Liu P, Zhang A, Xu Y, Xu LX. Study of non-uniform nanoparticle liposome extravasation in tumor. International Journal of Hyperthermia 2005; 21(12): 259-270.

19. Maier-Hauff K, Rothe R, Scholz R, Gneveckow U, Wust P, Thiesen B et al. Intracranial thermotherapy using magnetic nanoparticles combined with external beam radiotherapy: results of feasibility study on patients with glioblastoma multiforme. Journal of NeuroOncology 2007; 81(1): 53-60.

20. Maier-Hauff K, Ulrich F, Nestler D, Niehoff H, Wust P, Thiesen B et al. Efficacy and safety of intratumoral thermotherapy using magnetic iron-oxide nanoparticles combined with external beam radiotherapy on patients with recurrent glioblastoma multiforme. Journal of Neuro-Oncology 2011; 103(2): 317-324.

21. Manuchehrabadi N, Chen Y, LeBrun A, Ma R, Zhu L. Computational simulation of temperature elevation in tumors using Monte Carlo method and comparison to experimental measurements in laser photothermal Therapy. ASME Journal of Biomechanical Engineering 2013; 135(12): 121007.

22. Manuchehrabadi N, Zhu L. Development of a computational simulation tool to design a protocol for treating prostate tumours using transurethral laser photothermal therapy. International Journal of Hyperthermia 2014; 30(6): 349-361. 
23. Marqa MF, Colin P, Nevoux P, Mordon SR, Betrouni N. Focal laser ablation of prostate cancer: numerical simulation of temperature and damage distribution. Biomedical Engineering Online 2011; 10; 45.

24. McGuire S, Yuan F. Quantitative analysis of intratumoral infusion of color molecules. American Journal of Physiology 2001; 281(2): H715-H721.

25. Pearce JA. Improving accuracy in Arrhenius models to cell death: adding a temperaturedependent time delay. Journal of Biomechanical Engineering 2015; 137(12): 121006.

26. Pennes HH. Analysis of tissue and arterial blood temperatures in the resting forearm. Journal of Applied Physiology 1948; 1:93-122.

27. Ponce AM, Vujaskovic Z, Yuan F, Needham D, Dewhirst MW. Hyperthermia mediated liposomal drug delivery. International Journal of Hyperthermia 2006; 22(3) 205-213.

28. Rylander MN, Feng Y, Zhang Y, Bass J, Stafford RJ, Volgin A et al. Optimizing heat shock protein expression induced by prostate cancer laser therapy through predictive computational models. Journal of Biomedical Optics 2006; 11(4): 041113.

29. Trakic A, Lui F, Crozier S. Transient temperature rise in a mouse due to low-frequency regional hyperthermia. Physics in Medicine and Biology 2006; 51: 1673-1691.

30. Urono M, Kuroda M, Nishimura Y. For the clinical application of thermochemotherapy given at mild temperatures. International Journal of Hyperthermia 1999, 15(2): 79-107.

31. Wust P, Gneveckow U, Johannsen M, Böhmer D, Henkel T, Kahmann F et al. Magnetic nanoparticles for interstitial thermotherapy-feasibility, tolerance and achieved temperatures. International Journal of Hyperthermia 2006; 22(8): 673-685. 
32. Zhao Q, Wang L, Cheng R, Mao L, Arnold R, Howerth EW et al. Magnetic nanoparticle-based hyperthermia for head \& neck cancer in mouse models. Theranostics 2012; 2(1): 113-121.

33. Zhu L. Recent developments in biotransport. ASME Journal of Thermodynamic Sciences and Engineering Applications 2010; 2(4): 040801 
Tables

Table 1. Material, thermal, and physiological properties of PC3 tumors and tissue [Trakic et al., 2006]

\begin{tabular}{ccc}
\hline Parameter & Symbol & Value \\
\hline Density & $\rho_{t}, \rho_{c}, \rho_{b}$ & $1000\left(\mathrm{~kg} / \mathrm{m}^{3}\right)$ \\
\hline Specific Heat & $c_{t}, c_{c}, c_{b}$ & $3500(\mathrm{~J} / \mathrm{kg} \cdot \mathrm{K})$ \\
\hline Thermal Conductivity & $k_{t}, k_{c}$ & $0.642(\mathrm{~W} / \mathrm{m} \cdot \mathrm{K})$ \\
\hline Metabolic Heat Generation of Tissue & $Q_{m e t, t}$ & $25829\left(\mathrm{~W} / \mathrm{m}^{3}\right)$ \\
\hline Metabolic Heat Generation of Tumor & $Q_{m e t, c}$ & $2708\left(\mathrm{~W} / \mathrm{m}^{3}\right)$ \\
\hline Blood Perfusion Rate of Tissue & $\omega_{t}$ & $0.003(1 / \mathrm{s})$
\end{tabular}

Table 2. Arrhenius parameters for PC3 tumors [Rylander et al., 2006]

\begin{tabular}{ccccc}
\hline Parameter & Symbol & Temperature & PC3 Cells & Tissue \\
\hline Frequency factor & $A$ & $T \leq 54^{\circ} \mathrm{C}$ & $1.8 \times 10^{36}$ & $6.36 \times 10^{19}$ \\
$(\mathbf{1} / \mathbf{s})$ & & $T>54^{\circ} \mathrm{C}$ & $7 \times 10^{17}$ & - \\
\hline Activation energy & $E_{a}$ & $T \leq 54^{\circ} \mathrm{C}$ & $2.38 \times 10^{5}$ & $1.38 \times 10^{5}$ \\
$(\mathbf{J} / \mathbf{m o l})$ & & $T>54^{\circ} \mathrm{C}$ & $1.24 \times 10^{5}$ & - \\
\hline
\end{tabular}




\section{Figure legends:}

Figure 1. (a) A simplified mouse model with an implanted tumor and (b) the head-on view of the model.

Figure 2. The mouse model with the embedded tumor after meshing and the prescribed boundary conditions.

Figure 3. A reconstructed microCT image (a), its corresponding $S A R$ distribution (b) shown in COMSOL, and the simulated steady-state temperature distribution in COMSOL (c) based on that in a tumor injected with $0.1 \mathrm{cc}$ of a $5.8 \%$ ferrofluid at an infusion rate of $3 \mu \mathrm{L} / \mathrm{min}$.

Figure 4. Slices of the temperature fields in the tumor and mouse body: (a) before heating, (b) 3 minutes, (c) 6 minutes, (d) 9 minutes, (e) 12 minutes, and (f) 15 minutes after the heating starts. Note that the steady-state temperature field is established after 15 minutes.

Figure 5. Damage contours (a) before heating, (b) 5 minutes, (c) 12 minutes, (d) 15 minutes, (e) 20 minutes, and (f) 25 minutes after heating. The dark red line represents the $\Omega=4$ contour. The heavy white lines in (b) and (c) represent the $\Omega=1$ contour.

Figure 6. Damage contours in a single slice after the heating session of 25 minutes. The region of the collateral thermal damage to the healthy mouse tissue is shown in the shadowed pink region.

Figure 7. Effects of the infusion rate on the designed heating time, when either $\Omega=1$ or $\Omega=4$ is selected as the damage threshold.

Figure 8. Percent volume of collateral thermal damage to the surrounding healthy tissue after complete thermal treatment $(\Omega \geq 4)$. 
(a)

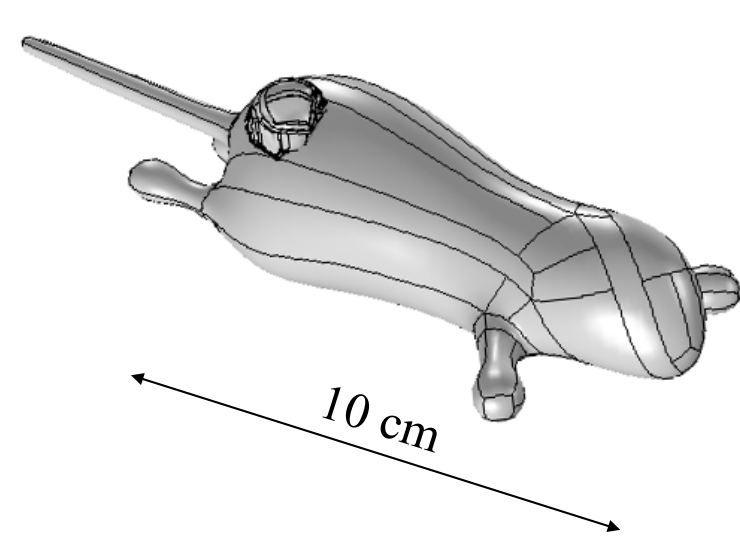

(b)

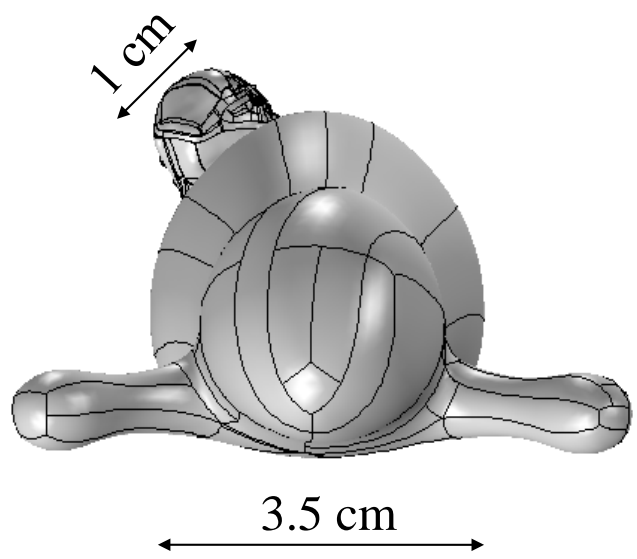

Figure 1. (a) A simplified mouse model with an implanted tumor and (b) the head-on view of the model. 


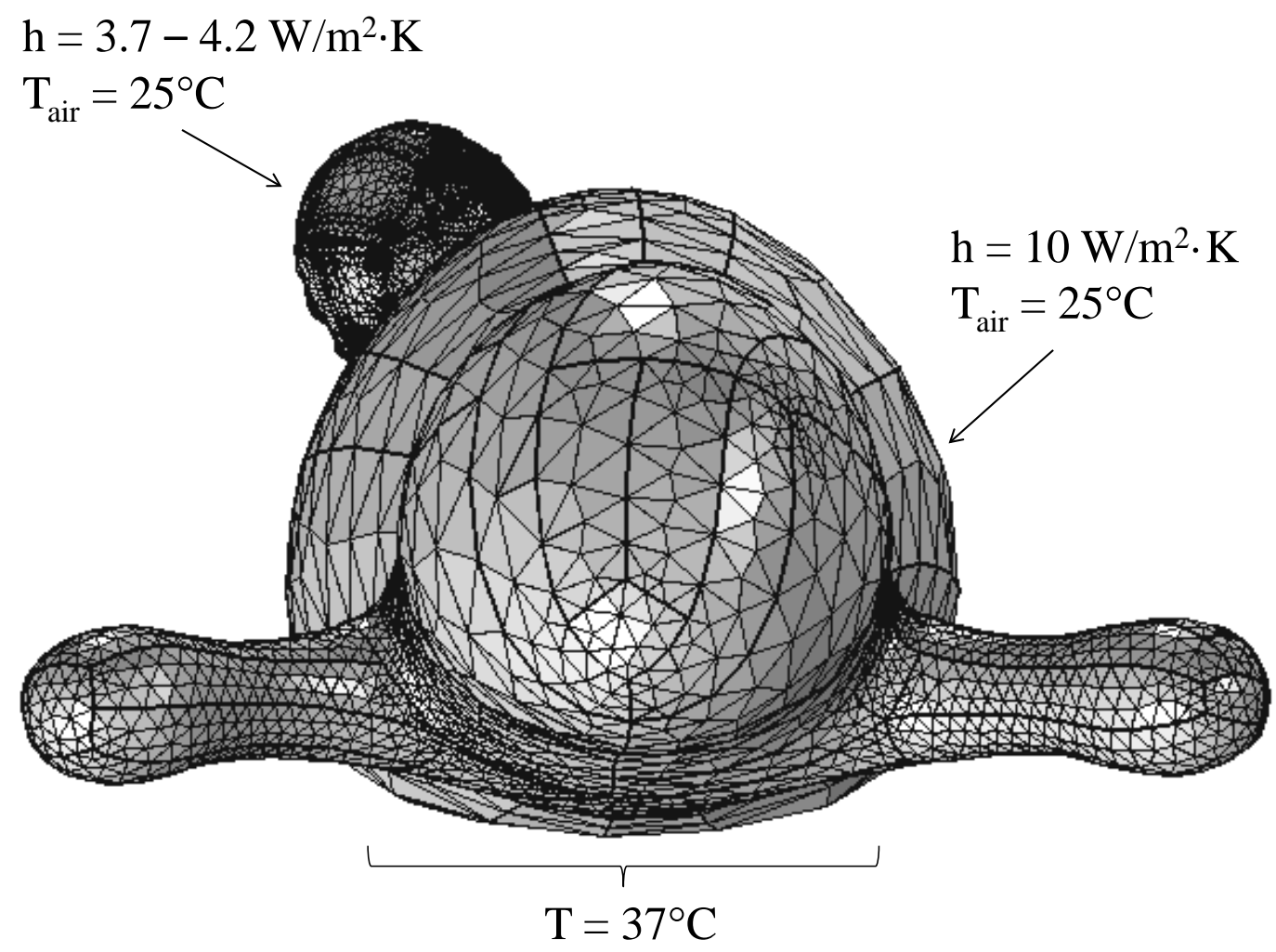

Figure 2. The mouse model with the embedded tumor after meshing and the prescribed boundary conditions. 


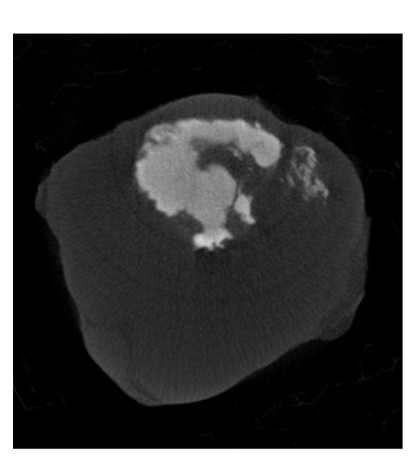

(a)

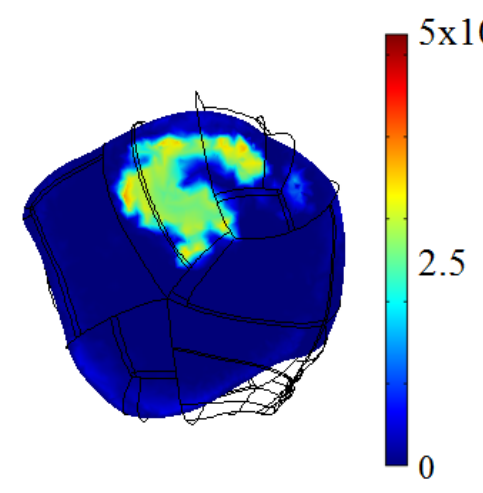

(b)

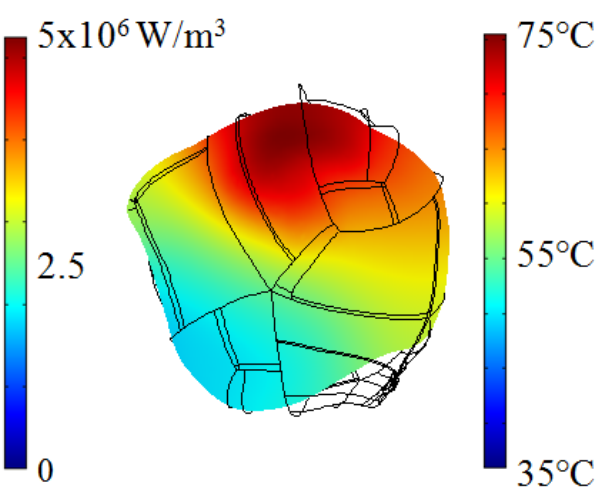

(c)

Figure 3. A reconstructed microCT image (a), its corresponding $Q_{M N H}$ distribution (b) shown in COMSOL, and the simulated steady-state temperature distribution in COMSOL (c) based on that in a tumor injected with $0.1 \mathrm{cc}$ of a $5.8 \%$ ferrofluid at an infusion rate of $3 \mu \mathrm{L} / \mathrm{min}$. 


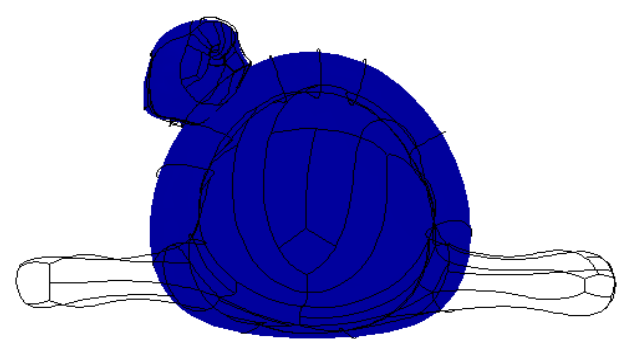

(a)

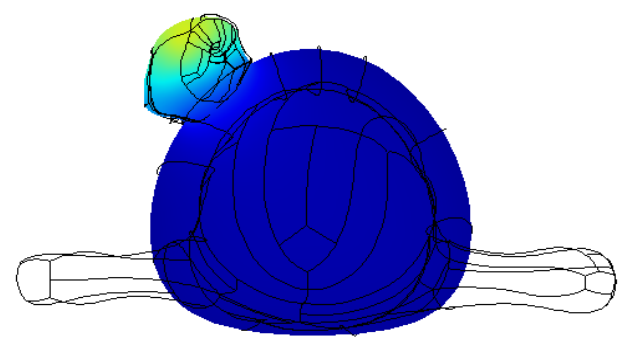

(c)

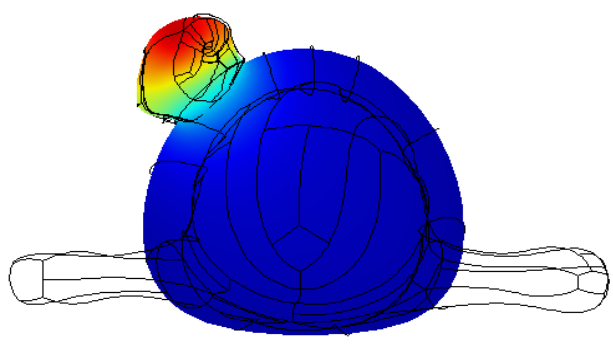

(e)

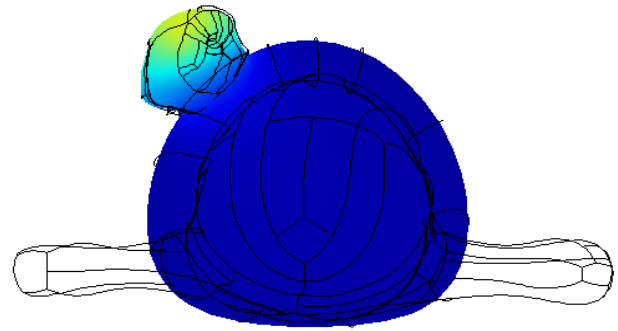

(b)

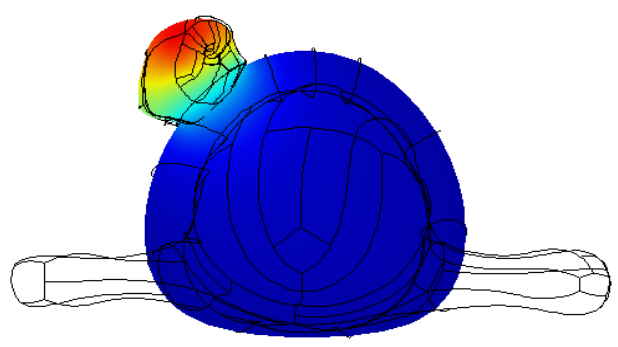

(d)

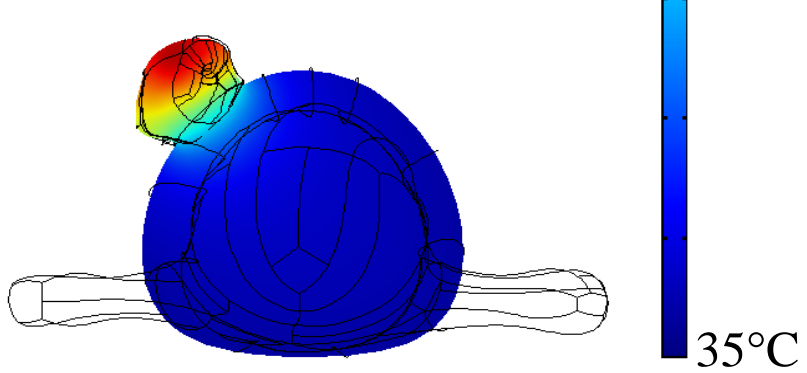

(f)

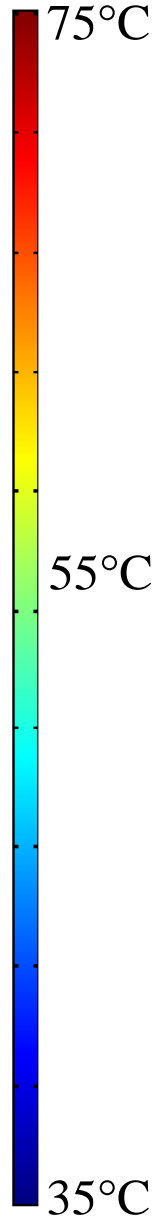

Figure 4. Slices of the temperature fields in the tumor and mouse body: (a) before heating, (b) 3 minutes, (c) 6 minutes, (d) 9 minutes, (e) 12 minutes, and (f) 15 minutes after the heating starts. Note that the steady-state temperature field is established after 15 minutes. 


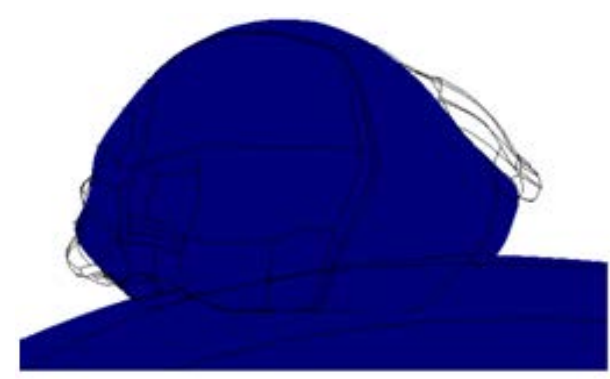

(a)

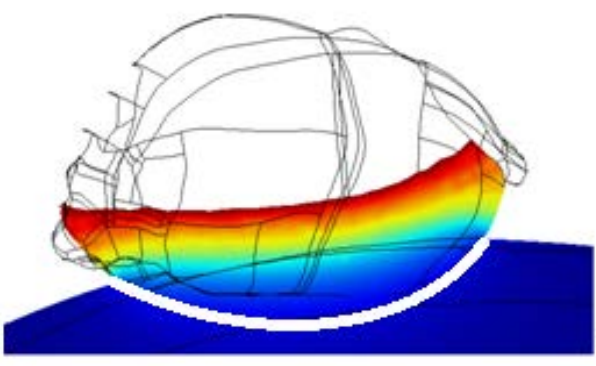

(c)

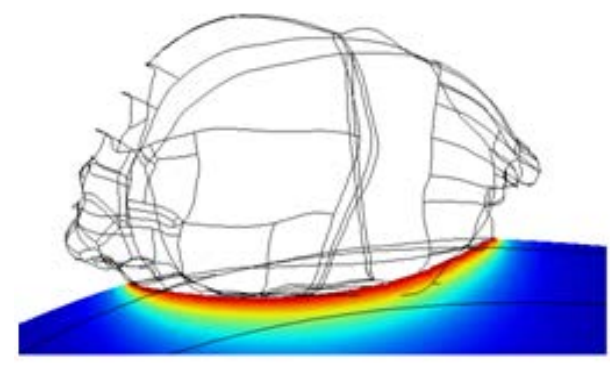

(e)

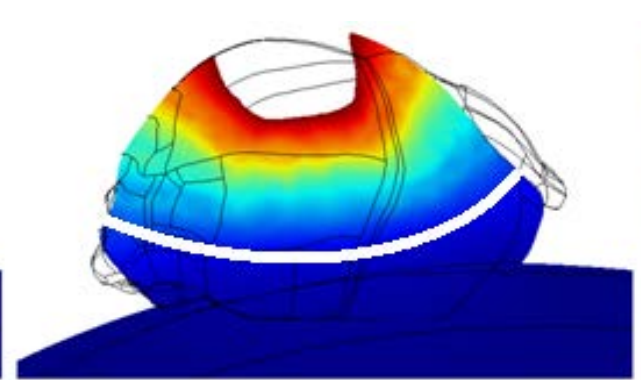

(b)

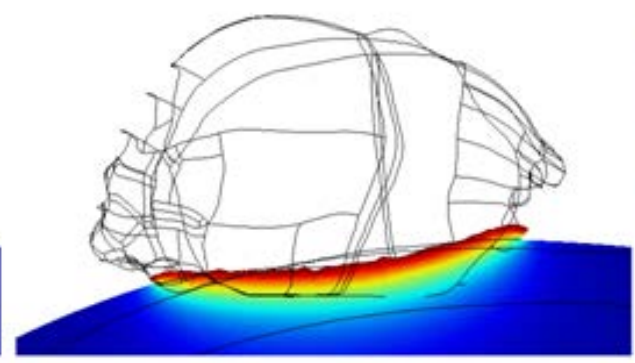

(d)

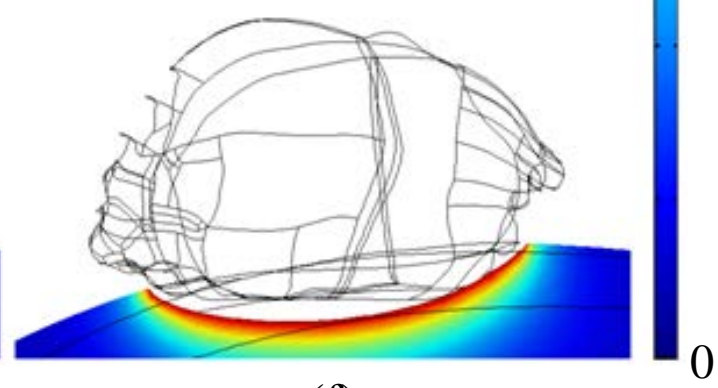

(f)

Figure 5. Evolution of damage contours (a) before heating, (b) 5 minutes, (c) 12 minutes, (d) 15 minutes, (e) 20 minutes, and (f) 25 minutes after heating. The dark red line represents the $\Omega=4$ contour. The heavy white lines in (b) and (c) represent the $\Omega=1$ contour. 


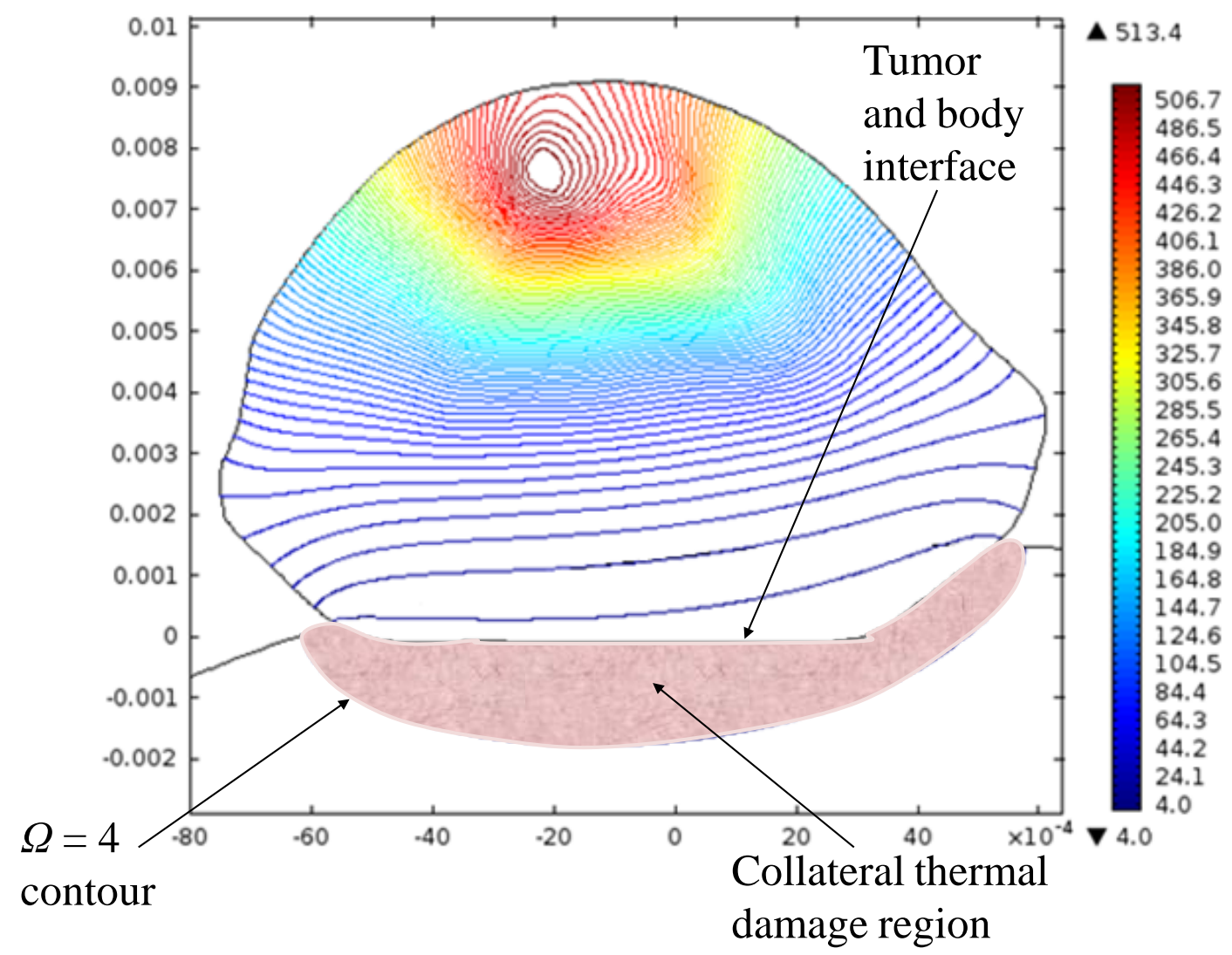

Figure 6. Damage contours in a single slice after the heating session of 25 minutes. The region of the collateral thermal damage to the healthy mouse tissue is shown in the shadowed pink region. 


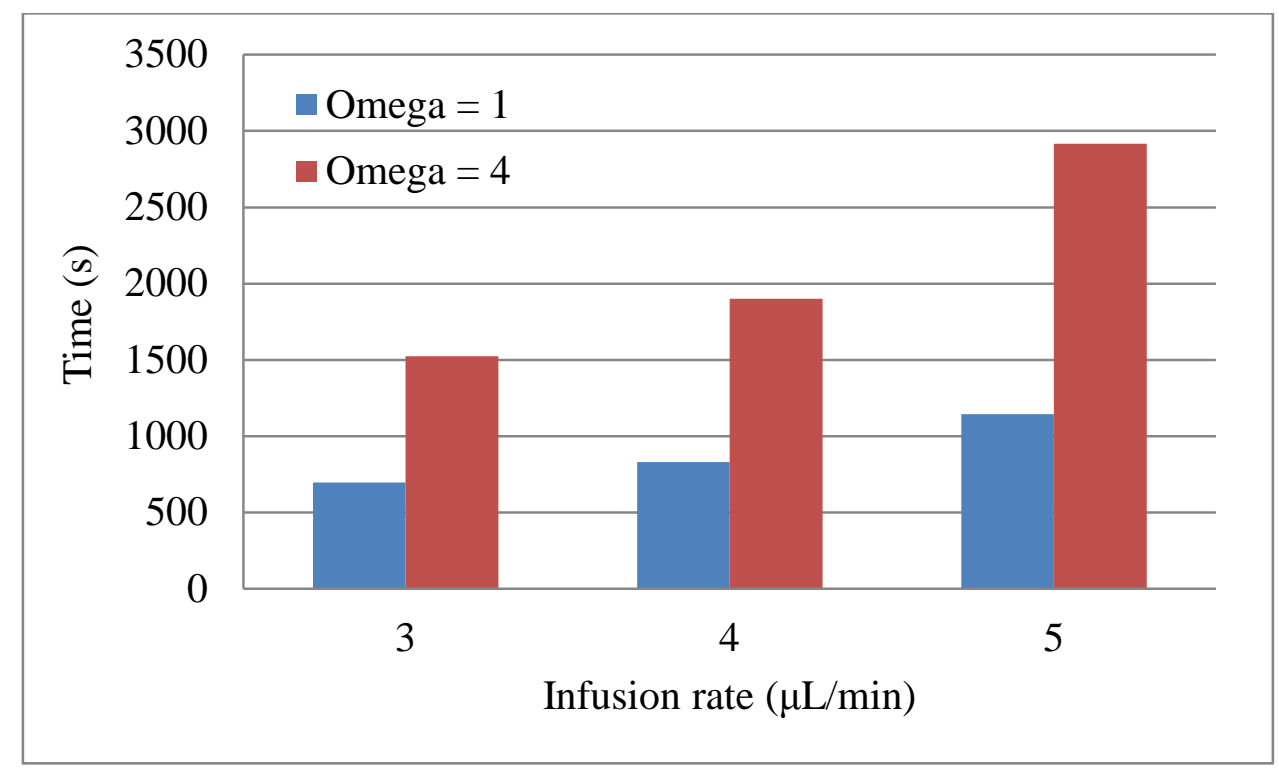

Figure 7. Effects of the infusion rate on the designed heating time, when either $\Omega=1$ or $\Omega=4$ is selected as the damage threshold. 


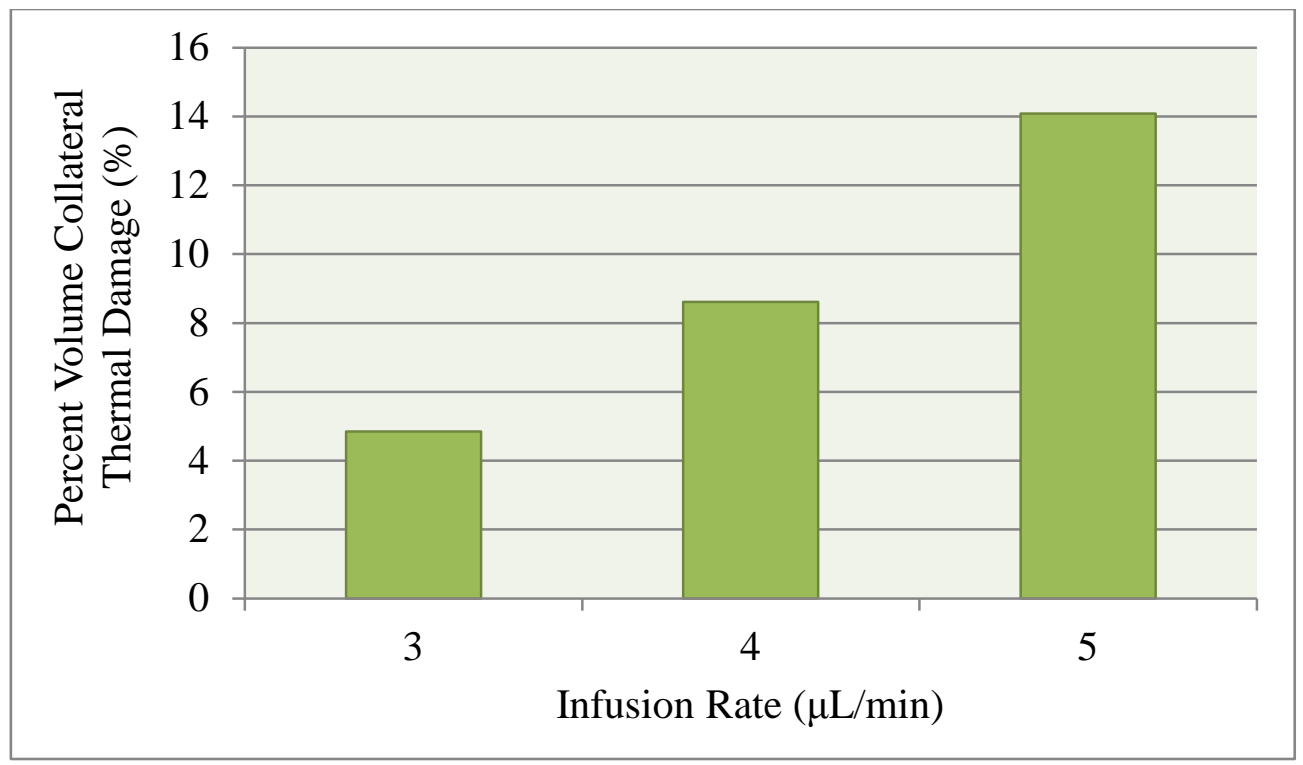

Figure 8. Percent volume of collateral thermal damage to the surrounding healthy tissue after complete thermal treatment $(\Omega \geq 4)$. 